\section{Signo del halo invertido como manifestación inhabitual de neumonía organizada criptogénica. Reporte de un caso}

\author{
GIANCARLO SCHIAPPACASSE ${ }^{1,7}$, ARTURO ACEVEDO ${ }^{3,4}$, \\ RENÉ MARTÍNEZ ${ }^{1,5}$, JORGE ESCOBAR $^{3,4,6}$, \\ ANTONIO HERNÁNDEZ², YUMAY PIRES ${ }^{7}$
}

\section{Reversed halo sign as an unusual manifestation of cryptogenic organized pneumonia. Report of one case}

We report a 64 years old female admitted with fever, cough, dyspnea and lung opacities in the chest X ray. A chest tomography scan (CTS) showed multiple-bilateral ring-shaped opacities and the reversed halo sign (RHS). The patient did not improve with antimicrobial therapy $(A T)$. Infection and rheumatologic causes were excluded, therefore Cryptogenic organizing pneumonia (COP) was suspected with compatible percutaneous biopsy. Systemic steroids were started with a good clinical response. The patient was discharged four weeks after admission in good general conditions and practically no lungs opacities.

(Rev Med Chile 2019; 147: 663-667)

Key words: Cryptogenic Organizing Pneumonia; Signs and Symptoms, Respiratory; Tomography.

$\mathrm{E}$ l signo del halo invertido se caracteriza por una opacidad central de vidrio esmerilado rodeada por una consolidación del espacio aéreo más densa en forma de una media luna o un anillo. Inicialmente esto fue descrito como un signo específico para la neumonía organizada criptogéni$\mathrm{ca}^{1,2}$, posteriormente, el signo del halo invertido se ha informado en asociación con una amplia gama de enfermedades pulmonares, de etiología benigna o maligna, en inmunocompetentes o inmunodeprimidos, incluidas las infecciones fúngicas pulmonares invasivas, para coccidioidomicosis, neumonía por Pneumocystis jirovecii, tuberculosis, neumonía adquirida en la comunidad, granulomatosis linfomatoidea, granulomatosis de Wegener, neumonía lipoidea y sarcoidosis ${ }^{1,2,4}$. También se puede observar en neoplasias pulmonares e infarto, luego de radioterapia y ablación por radiofrecuencia de neoplasias malignas pulmonares ${ }^{2}$.
'Servicio de Radiología de

Hospital Militar de Santiago.

${ }^{2}$ Servicio de Neumología de

Hospital Militar de Santiago.

${ }^{3}$ Servicio de Medicina Interna de

Hospital Militar de Santiago.

${ }^{4}$ Escuela de Postgrado de

Medicina de Universidad de los

Andes.

${ }^{5}$ Residente de Radiología

Universidad de Santiago.

${ }^{6}$ Residente de Medicina Interna

Universidad de los Andes.

${ }^{7}$ Facultad de Medicina

Universidad del Desarrollo Clínica

Alemana de Santiago.

Trabajo no recibió

financiamiento.

Los autores declaran no tener conflictos de interés.

Recibido el 5 de noviembre de 2018, aceptado el 23 de mayo de 2019.

Correspondencia a:

Giancarlo Schiappacasse Departamento de imágenes Hospital Militar de Santiago. Clínica Alemana de Santiago. gschiappacasse@gmail.com
Se presenta a continuación un caso clínico de neumonía organizada con el signo del halo invertido como manifestación radiológica a destacar. La neumonia organizada es una entidad bien definida, con diversas características imagenológicas, puede obedecer a numerosas patologías, adquiriendo el carácter de criptogénica cuando no se encuentra una causa. Entre las manifestaciones radiológicas, el signo del halo invertido se observa solo en una quinta parte de los pacientes con la enfermedad, parece ser relativamente específico para realizar un diagnóstico de neumonía organizada criptogénica en la tomografía computada (TC) ${ }^{3,4}$.

\section{Caso clínico}

Paciente de sexo femenino de 64 años de edad. Refiere cuadro de 7 días de evolución caracteri- 
zado por fiebre de hasta $39^{\circ} \mathrm{C}$ y tos seca asociado a disnea progresiva y compromiso de estado general. Consulta a servicio de urgencias, donde se interpreta neumonía adquirida en la comunidad, indicando tratamiento en domicilio bajo cobertura antibiótica con amoxicilina y ácido clavulánico. Ante la respuesta desfavorable, decide consultar nuevamente al servicio de urgencias donde ingresa taquicárdica (120 latidos por minuto) y febril $\left(38^{\circ} \mathrm{C}\right)$; resto de los signos vitales sin hallazgos. Al examen físico sólo destacaban crépitos en las bases pulmonares. Se solicita Rx de tórax evidenciando múltiples opacidades redondeadas circunscritas de centro radiolúcido y de tamaño variable en parénquima pulmonar (Figura 1). En los exámenes de laboratorio al ingreso destacaban elevación de la PCR a $191 \mathrm{mg} / \mathrm{dL}$ con un recuento de leucocitos normal (Tabla 1).

Las imágenes descritas inicialmente se interpretaron como posible presencia de abscesos pulmonares, por lo que se decide ingreso hospitalario para estudio y manejo, en sala de cuidados generales de medicina interna.

Al ingreso se realiza TC de tórax, constatándose

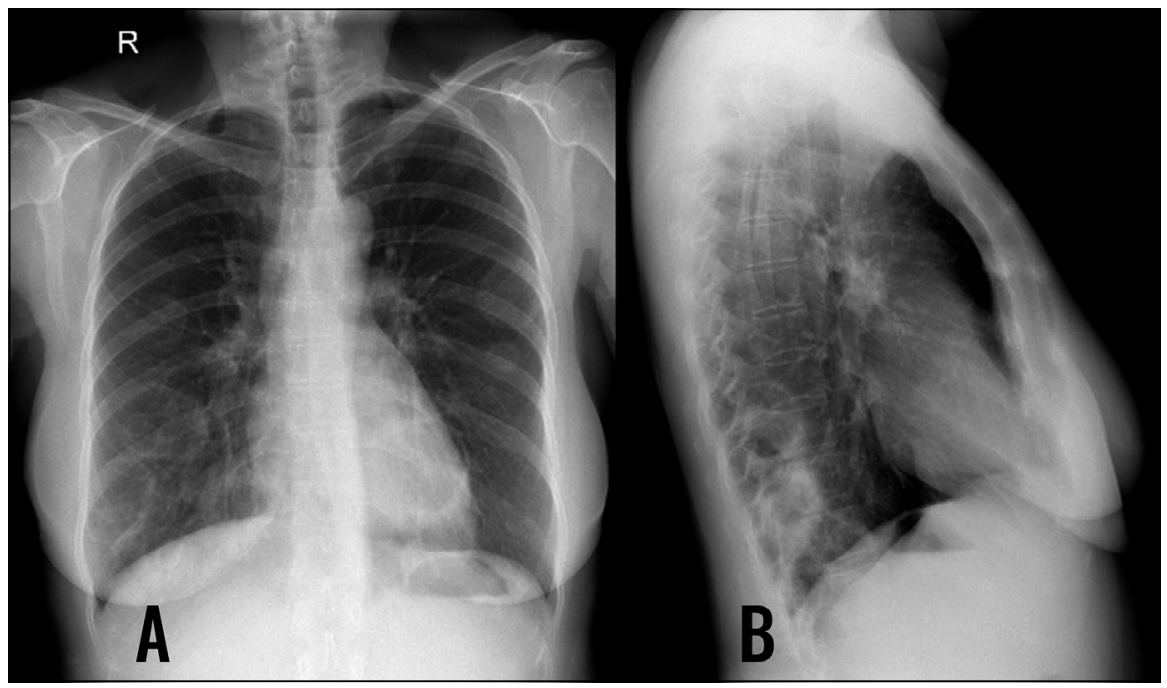

Figura 1. A-B: Radiografía de tórax posteroanterior y lateral, donde se aprecian múltiples opacidades parenquimatosas pulmonares difusas.

Tabla 1. Exámenes de laboratorio

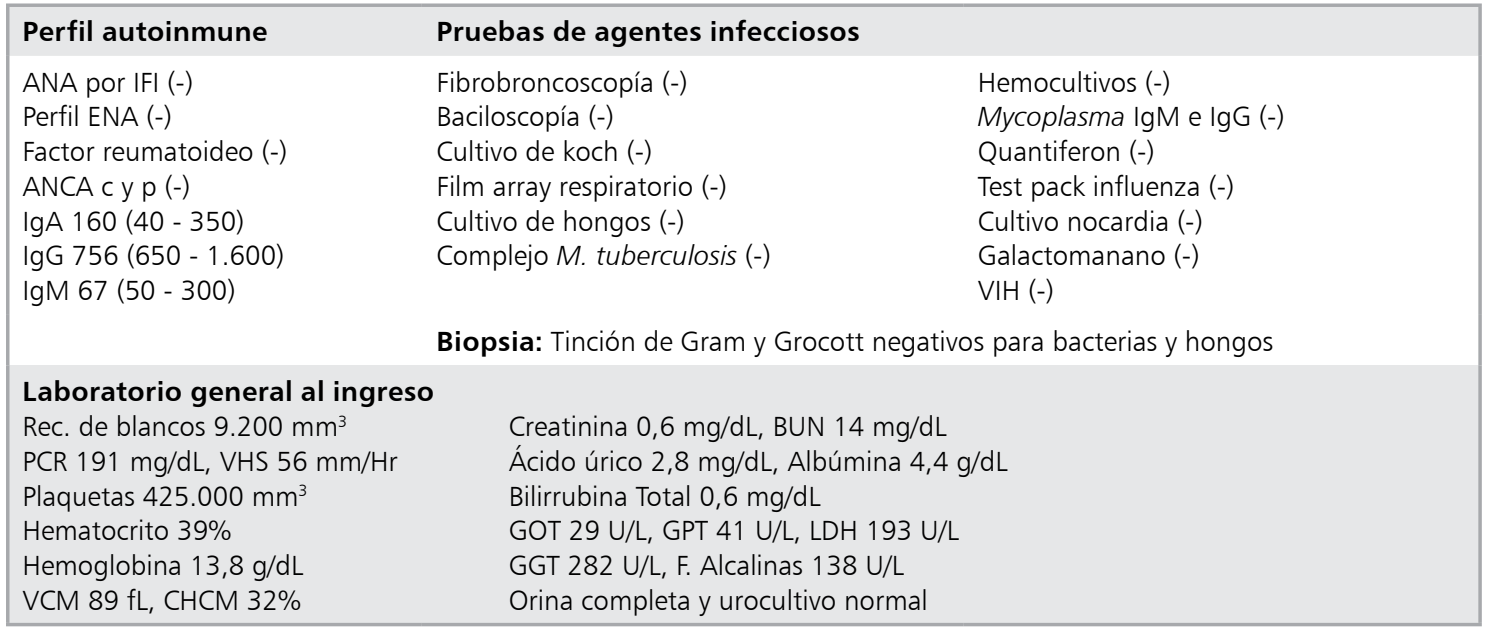


múltiples opacidades en vidrio esmerilado rodeadas por halo condensante, hallazgos interpretados como signo del halo invertido (Figura 2).

Se indicó ceftriaxona por 7 días asociado a oseltamivir y azitromicina por 5 días, persistiendo febril sin mejoría del cuadro. Se evalua por infectología, indicándose estudio complementario a fin de descartar etiologías del signo del halo invertido (Tabla 1). Se interroga dirigídamente, sin antecedentes personales ni familiares de enfermedad autoinmune, ni clínica sugerente de enfermedad tejido conectivo ni vasculitis. El perfil autoinmune es negativo. Se evalua por reumatología quienes toman la decisión de no ampliar el estudio de posible etiología reumatológica.

Tras la persistencia del cuadro clínico sin identificar etiología, se indica la realización de biopsia por punción transtorácica por radiología intervencional. Posterior a procedimiento, el paciente presenta hemoptisis, requiriendo intubación profiláctica y monitoreo en unidad de cuidados intensivos, con corta estadía tras evolución favorable. Se rescata resultado de biopsia, la cual informa hallazgos compatibles con neumonía organizada (Figuras 3 y 4), sin visualización de bacterias ni hongos en tinciones específicas (Tabla 1).

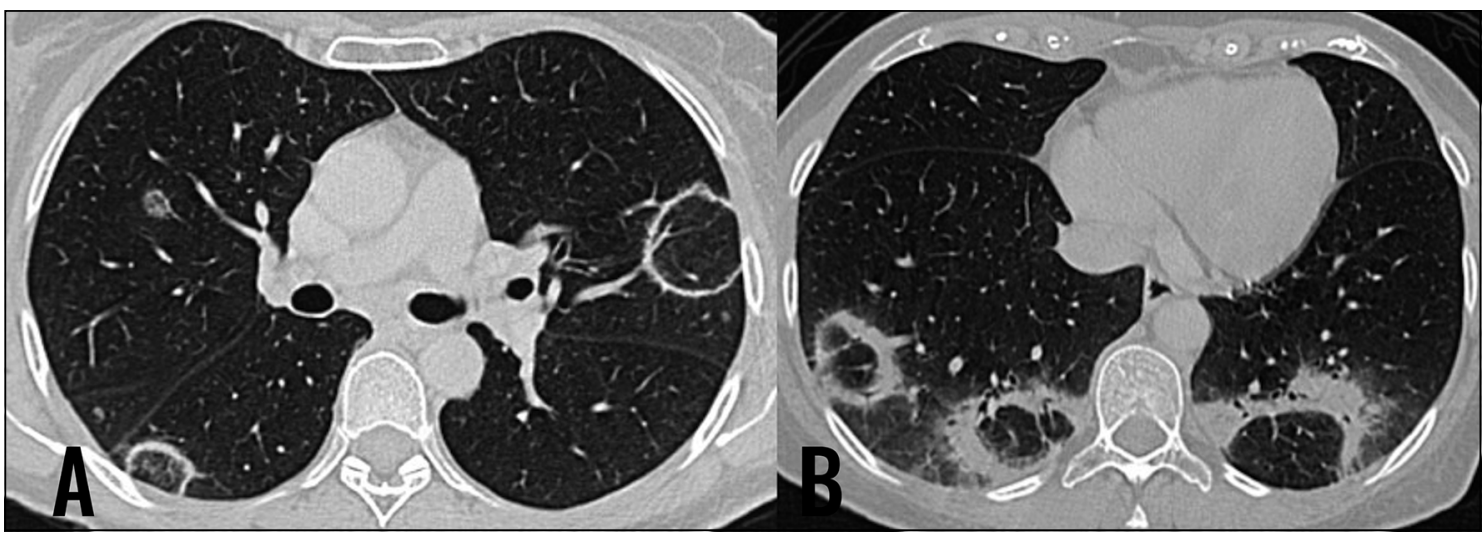

Figura 2. A-B: Tomografía computarizada de tórax con contraste, en la cual se evidencian numerosas áreas en vidrio esmerilado de distribución difusa, rodeado por consolidaciones en forma de anillo (signo del halo invertido), que en los lóbulos inferiores tienden a confluir.

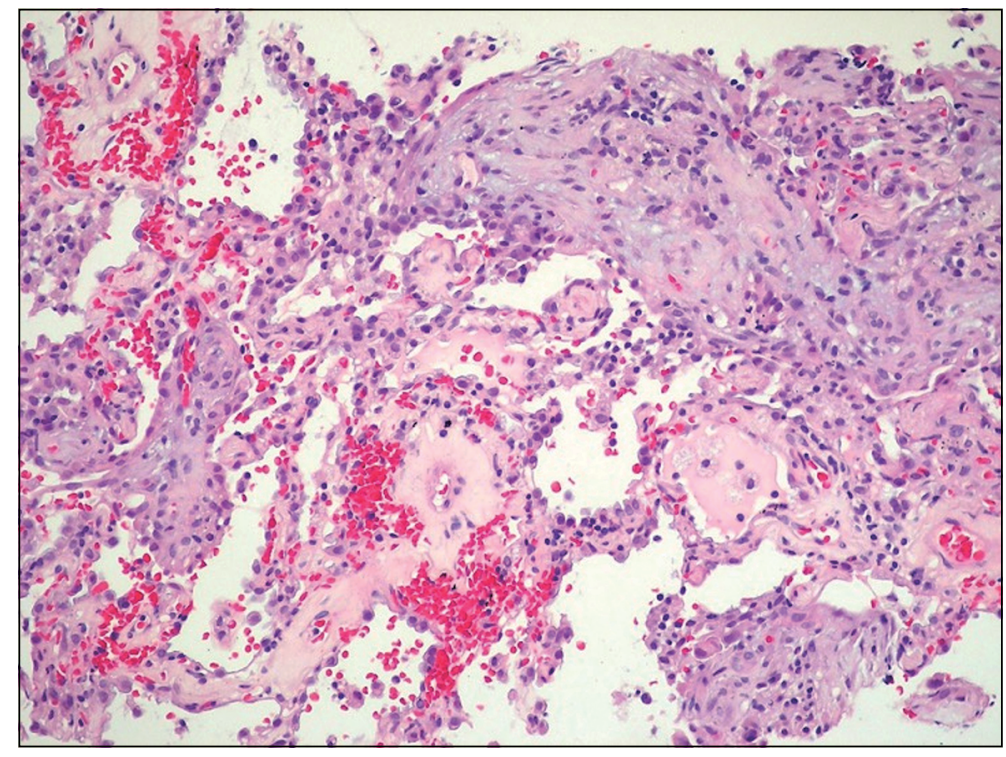

Figura 3. Corte histológico de tejido pulmonar. Tinción con hematoxilina y eosina. Muestra leve ensanchamiento septal, escaso infiltrado inflamatorio predominantemente linfocitario intersticial y tapones de tejido conectivo laxo en algunos espacios alveolares. 


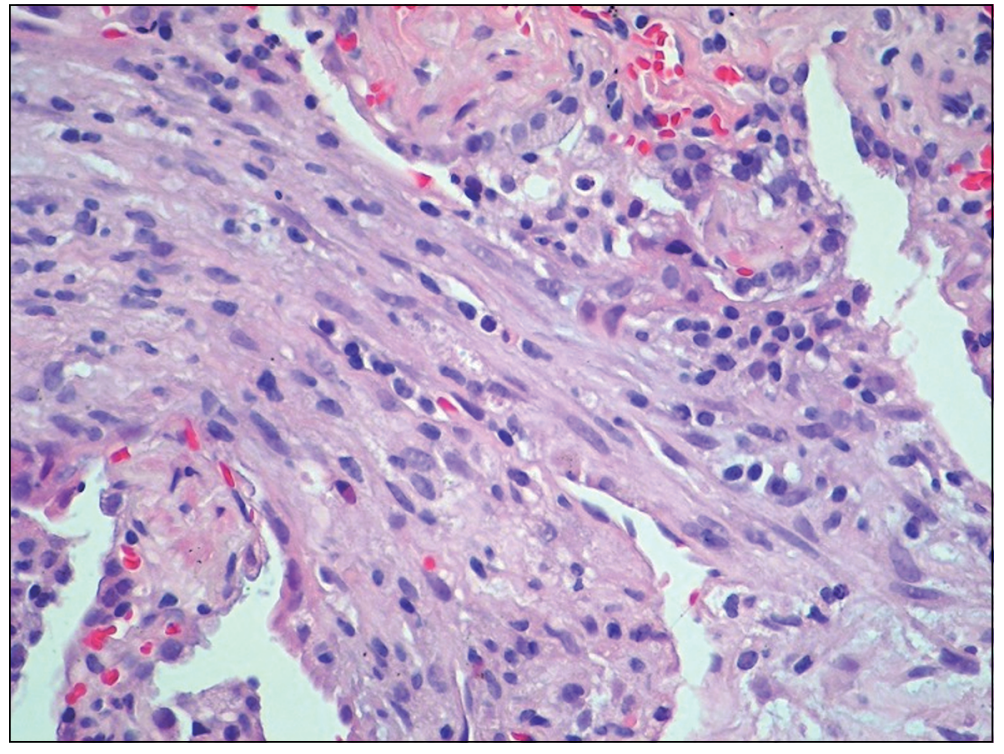

Figura 4. Corte histológico de tejido pulmonar. Tinción con hematoxilina y eosina. Muestra tapón de tejido conectivo laxo intraalveolar con mayor detaIle, constituido por células fusiformes fibroblásticas y con células inflamatorias dispersas.

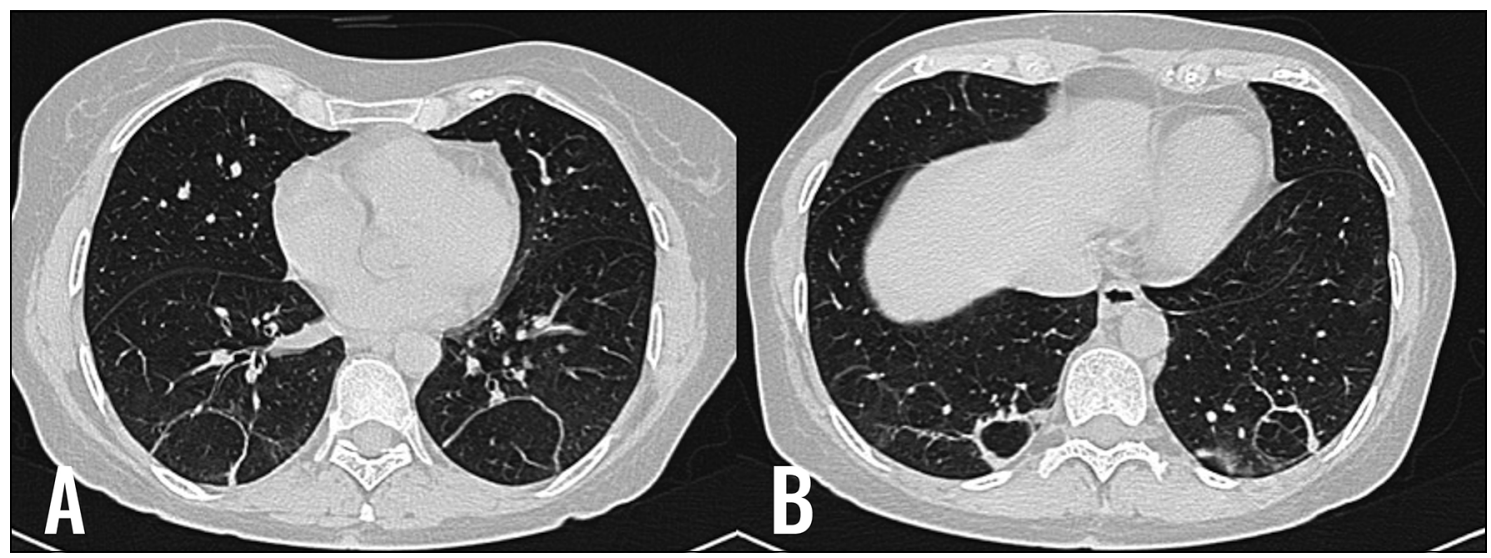

Figura 5. A-B: Tomografía computarizada de tórax sin contraste, que demuestra regresión significativa de todas las lesiones pulmonares, con desaparición de las más pequeñas, identificándose solo finas opacidades lineales residuales de morfología redondeada.

Se diagnostica neumonía organizada y dada la ausencia de etiología se le atribuye un origen criptogénico, indicándose terapia esteroidal empírica con prednisona $1 \mathrm{mg} / \mathrm{kg} /$ día. Tras el inicio de éste, se logra buena respuesta, con un cuadro respiratorio en remisión, sin nuevos peaks febriles y parámetros inflamatorios en descenso hasta su normalización, además una regresión practicamente completa de las consolidaciones anulares bilaterales (Figura 5). Se decide egreso hospitalario para continuar manejo de manera ambulatoria.

\section{Discusión}

El caso clínico presentado presenta cierta complejidad para el equipo médico en lograr una hipótesis diagnóstica y en consecuencia un afrontamiento terapéutico adecuado. Las etiologías infecciosa y autoinmune también fueron desestimadas tanto por infectología como reumatología, tras completar el estudio de manera dirigida, además de la refractariedad a la terapia antibiótica. Tampoco tenía antecedentes de síndrome consuntivo, "síntomas B", ni alteraciones 
en exámenes de laboratorio que pudieran orientar a causas neoplásicas. Para completar el estudio, se realiza biopsia percutánea, que evidencia hallazgos compatibles con neumonía organizada (Figuras 3 y 4). Se diagnostica finalmente neumonía organizada criptogénica, tras descartar causas secundarias y la excelente respuesta clínica al tratamiento con prednisona $60 \mathrm{mg}$ al día, logrando resolución de la sintomatología respiratoria, normalización de los parámetros inflamatorios y regresión de las lesiones pulmonares (Figura 5).

Las imágenes presentadas en la tomografía computarizada de tórax implican una amplia gama de diagnósticos diferenciales para la pneumonia organizada y el signo del halo invertido, hallazgos que si bien son característicos, no son específicos ${ }^{5-8}$.

Primero es necesario determinar si el paciente es inmunocompetente o inmunocomprometido. Por ejemplo, en inmunocomprometidos con neumonia organizada y el signo del halo invertido secundario a infección fúngica invasora, son pacientes con un marcado deterioro del estado general y compromiso ventilatorio severo, contexto clínico completamente distinto al caso descrito ${ }^{9-11}$.

Hay que considerar el error muestral que ofrece la biopsia por vía percutánea ${ }^{12}$, lo cual fue evaluado y discutido como un factor que podría retardar e incluso limitar el diagnóstico definitivo. En este caso se obtuvo una buena calidad de muestra y no hubo duda alguna en los hallazgos descritos por anatomopatología (Figuras 3 y 4 ).

Los indicadores más específicos de neumonía organizada son alteraciones perilobulares, signo de halo inverso, bandas radiales de consolidación que contienen un broncograma aéreo con migración de las lesiones a lo largo del tiempo mientras que las lesiones tumorales son estacionarias. Además, el engrosamiento de los ganglios linfáticos y el líquido pleural rara vez se observan en la OP, pero a menudo se informan en el carcinoma de pulmón $^{12}$.

Fue fundamental la correlación clínico radiológica para plantear el diagnóstico diferencial y lograr llegar a un diagnóstico definitivo, ya que al ser una patología infrecuente requiere de un equipo multidisciplinario ${ }^{13}$.

\section{Referencias}

1. Marchiori, Edson \& Hochhegger, Bruno \& Zanetti, Gláucia. (2016). Reversed halo sign in invasive fungal infections. Jornal Brasileiro de Pneumologia. 42. 232232. 10.1590/S1806-37562016000000119.

2. Godoy MC, Viswanathan C, Marchiori E, Truong MT, Benveniste MF, Rossi S. The reversed halo sign: Update and differential diagnosis. Br J Radiol 2012; 85: 1226 35.

3. Kim SJ, Lee KS, Ryu YH. Reversed halo sign on high-resolution CT of cryptogenic organizing pneumonia: diagnostic implications. AJR 2003; 180: 1251-4.

4. Gasparetto EL, Escuissato DL, Davaus T. Reversed halo sign in pulmonary paracoccidioidomycosis. AJR 2005; 184: 1932-4.

5. Agarwal R, Aggarwal AN, Gupta D. Another cause of reverse halo sign: Wegener's granulomatosis. Br J Radiol 2007; 80 (958): 849-50.

6. Ahuja A, Gothi D, Joshi JM. A 15-year-old boy with "reversed halo". Indian J Chest Dis Allied Sci 2007; 49: 99-101.

7. Arai T, Inoue $\mathrm{Y}$, Ando S, Inoue K, Tsuyuguchi K, Suzuki K. [A case of cryptogenic organizing pneumonia showing reversed halo sign on computed tomography of the chest]. Nihon Kokyuki Gakkai Zasshi 2007; 45 (8): 621-6. Article in Japanese.

8. Wahba H, Truong MT, Lei X, Kontoyiannis DP, Marom EM. Reversed halo sign in invasive pulmonary fungal infections. Clin Infect Dis 2008; 46 (11): 1733-7.

9. Bräunlich J, Seyfarth HJ, Gessner C, Gradistanac T, Wirtz H. Lymphomatoid granulomatosis: a short description of an unusual case of the disease. Pneumologie 2009; 63 (12): 697-701.

10. Marom EM, Kontoyiannis DP. Imaging studies for diagnosing invasive fungal pneumonia in immunocompromised patients. Curr Opin Infect Dis 2011; 24 (4): 309-14.

11. Georgiadou SP, Sipsas NV, Marom EM, Kontoyiannis DP. The diagnostic value of halo and reversed halo signs for invasive mold infections in compromised hosts. Clin Infect Dis 2011; 52 (9): 1144-55.

12. Ding QL, Lv D, Wang BJ, Zhang QL, Yu YM, Sun SF, et al. Macrolide therapy in cryptogenic organizing pneumonia: A case report and literature review. Exp Ther Med 2015; 9: 829-34. doi: 10.3892/etm.2015.2183.

13. American Thoracic Society, European Respiratory Society. American Thoracic Society/European Respiratory Society international multidisciplinary consensus classification of the idiopathic interstitial pneumonias. This joint statement of the American Thoracic Society (ATS), and the European Respiratory Society (ERS) was adopted by the ATS board of directors, June 2001 and by the ERS Executive Committee, June 2001. Am J Respir Crit Care Med 2002; 165 (2): 277-304. 